\title{
Criteria for spectral classification of cool stars using high-resolution spectra
}

\author{
David Montes ${ }^{1}$, Raquel M. Martínez-Arnáiz ${ }^{1}$, Jesus Maldonado ${ }^{1,2}$, \\ Juan Roa-Llamazares ${ }^{1}$, Javier López-Santiago ${ }^{1,3}$, \\ Inés Crespo-Chacón ${ }^{1}$ and Enrique Solano ${ }^{4}$ \\ ${ }^{1}$ Departamento de Astrofísica, Facultad de Ciencias Físicas, \\ Universidad Complutense de Madrid, E-28040 Madrid, Spain \\ email: dmg@astrax.fis.ucm.es \\ ${ }^{2}$ Departamento de Física Teórica, C-XI, Facultad de Ciencias, \\ Universidad Autónoma de Madrid, Cantoblanco, Spain \\ ${ }^{3}$ INAF - Osservatorio Astronomico di Palermo Giuseppe S. Vaiana, \\ Piazza Parlamento 1, I-90134, Palermo, Italy \\ email: jlopez@astropa.unipa.it \\ ${ }^{4}$ INTA - Laboratorio de Astrofísica Espacial y Física Fundamental, \\ P.O. Box 50727, E-28080 Madrid, Spain \\ email: esm@laeff.inta.es
}

We have compiled a large number of optical spectra of cool stars taken with different high-resolution echelle spectrographs $(R \simeq 40000)$. Many of those are available as spectral libraries (Montes et al. 1997, 1998, 1999, <http://www.ucm.es/info/Astrof/invest/ actividad/spectra.html) >.

We intend to include all these spectra in the Virtual Observatory (VO) following the standards of the International Virtual Observatory Alliance (IVOA).

The many VO tools that are or will be ready for the astrophysical community will make easier the use of these spectra in many areas, such as the study of chromospheric activity, spectral classification, determination of atmospheric parameters $\left(\mathrm{T}_{\text {eff }}, \log g,[\mathrm{Fe} / \mathrm{H}]\right)$, modeling stellar atmospheres, spectral synthesis applied to composite systems, and spectral synthesis of stellar population of galaxies.

In this contribution, as an example of the potential use of these spectra, we describe different spectral classification criteria for the cool stars (F5 to M5) based on equivalent width and equivalent width ratios of several photospheric lines, which are sensitive to effective temperature and luminosity class. To calibrate these relationships we have used a large number of optical spectra of spectral type standard stars (Morgan and Keenan (MK) standards) taken with different echelle spectrographs of similar spectral resolution. In addition, we have tested the behaviour of the photospheric lines with temperature by using synthetic spectra of main sequence stars $\left(\mathrm{T}_{\text {eff }}=6500-3500 \mathrm{~K}\right)$ with solar abundance computed using the ATLAS9 code by Kurucz (Kurucz, 1993), adapted to work under linux platform by Sbordone et al. (2004) and Sbordone (2005).

We describe in detail the behaviour of the equivalent width of photospheric lines like Fe I $\lambda 6430$, CaI $\lambda 6439$, and TiI $\lambda 5866$. Some equivalent width ratios as FeI $\lambda 6430 /$ FeII $\lambda 6432$, and FeI $\lambda 4071 /$ SrII $\lambda 4077$ and broad indices like MI6 $(\lambda 5125-5245) /(\lambda 5245-5290)$ are specially useful to determine effective temperature and discriminate between dwarf and giant stars.

The use of additional high-resolution spectra of MK standard stars throughout the VO will be very useful to improve these calibrations and obtain good criteria for spectral classification. 